Research Paper

\title{
Neuraminidase 1 deficiency attenuates cardiac dysfunction, oxidative stress, fibrosis, inflammatory via AMPK-SIRT3 pathway in diabetic cardiomyopathy mice
}

\author{
Zhen Guo ${ }^{1,2^{*}}$, Hu Tuo ${ }^{1,2^{*}}$, Nan Tang $3,4^{*}$, Fang-Yuan Liu ${ }^{1,2^{*}}$, Shu-Qing Ma1,2, Peng An ${ }^{1,2}$, Dan Yang ${ }^{1,2}$, Min-Yu \\ Wang ${ }^{1,2}$, Di Fan ${ }^{1,2 \bowtie}$, Zheng Yang ${ }^{1,2 \bowtie}$, Qi-Zhu Tang ${ }^{1,2}$ \\ 1. Department of Cardiology, Renmin Hospital of Wuhan University, Wuhan 430060, RP China \\ 2. Hubei Key Laboratory of Metabolic and Chronic Diseases, Wuhan, RP China \\ 3. The Affiliated Suqian Hospital of Xuzhou Medical University, Suqian 223800, RP China \\ 4. People's Hospital affiliated to Nanjing Drama Tower Hospital Group, Suqian 223800, RP China \\ *These authors contributed equally to this work. \\ Z.G, H.T, N.T, and FY.L contributed equally. \\ $\square$ Corresponding authors: Di Fan, Department of Cardiology, Renmin Hospital of Wuhan University, Cardiovascular Research Institute, Hubei Key Laboratory \\ of Metabolic and Chronic Diseases, Wuhan University at Jiefang Road 238, Wuhan 430060, RP China. Tel.: +86 27 88073385. Email: drfanti@yeah.net. Zheng \\ Yang, Department of Cardiology, Renmin Hospital of Wuhan University, Cardiovascular Research Institute, Hubei Key Laboratory of Metabolic and Chronic \\ Diseases, Wuhan University at Jiefang Road 238, Wuhan 430060, RP China. Tel.: +86 27 88073385. Email: 63376996@qq.com.Qi-Zhu Tang, Department of \\ Cardiology, Renmin Hospital of Wuhan University, Cardiovascular Research Institute, Hubei Key Laboratory of Metabolic and Chronic Diseases, Wuhan \\ University at Jiefang Road 238, Wuhan 430060, RP China. Tel.: +86 27 88073385. Email: qztang@whu.edu.cn.
}

(c) The author(s). This is an open access article distributed under the terms of the Creative Commons Attribution License (https://creativecommons.org/licenses/by/4.0/). See http://ivyspring.com/terms for full terms and conditions.

Received: 2021.08.10; Accepted: 2021.11.24; Published: 2022.01.01

\begin{abstract}
Diabetic cardiomyopathy (DCM) is associated with oxidative stress and augmented inflammation in the heart. Neuraminidases (NEU) 1 has initially been described as a lysosomal protein which plays a role in the catabolism of glycosylated proteins. We investigated the role of NEUI in the myocardium in diabetic heart. Streptozotocin (STZ) was injected intraperitoneally to induce diabetes in mice. Neonatal rat ventricular myocytes (NRVMs) were used to verify the effect of shNEU1 in vitro. NEU1 is up-regulated in cardiomyocytes under diabetic conditions. NEUI inhibition alleviated oxidative stress, inflammation and apoptosis, and improved cardiac function in STZ-induced diabetic mice. Furthermore, NEUI inhibition also attenuated the high glucose-induced increased reactive oxygen species generation, inflammation and, cell death in vitro. ShNEU1 activated Sirtuin 3 (SIRT3) signaling pathway, and SIRT3 deficiency blocked shNEU1-mediated cardioprotective effects in vitro. More importantly, we found AMPKa was responsible for the elevation of SIRT3 expression via AMPKa-deficiency studies in vitro and in vivo. Knockdown of LKB1 reversed the effect elicited by shNEU1 in vitro. In conclusion, NEU1 inhibition activates AMPKa via LKB1, and subsequently activates sirt3, thereby regulating fibrosis, inflammation, apoptosis and oxidative stress in diabetic myocardial tissue.
\end{abstract}

Key words: Neuraminidase 1; Diabetic cardiomyopathy; SIRT3; LKB1; AMPKa

\section{Introduction}

Diabetic cardiomyopathy $(\mathrm{DCM})$ is more common than clinically recognized. It is an important cause of heart failure in the world, and an essential burden of medical expenses in the whole country. Epidemiological studies have recognized that diabetes is an independent risk factor for cardiac dysfunction, characterized by myocardial hyper- trophy and diastolic/systolic dysfunction [1]. The pathophysiology of DCM is complex and despite significant advances, many fields are still not well understood. DCM has a complex pathogenic basis and is therefore difficult to treat by targeting a single candidate mechanism. In briefly, the mechanism of DCM involves increased oxidative/nitrosative stress 
[2], abnormal calcium signals [3], disordered glucose/ fatty acid metabolism [4, 5] and inflammatory pathways[6] lead to a variety of abnormal biochemical pathways, such as myocardial fibrosis[7], stiffness and hypertrophy [8], leading to potential pathological changes. These clinical effects develop from asymptomatic diastolic dysfunction to systolic dysfunction and clinical HF.

Neuraminidases (NEUs) are glycosidases, also known as sialidases, that catalyze the removal of a-glucoside-linked sialic acid residues from carbohydrate groups of glycoproteins and glycolipids. Four species of NEUs have been identified according to their subcellular localization and enzymological characteristics, namely NEU1, NEU2, NEU3, and NEU4. In addition to the typical catabolism function in lysosomes, NEU1 can also be transported to the cell surface and participate in the structure and function regulation of cell receptors. Some examples of receptors and cell signaling modulated by NEU1 salivation on the membrane include TLR4-NFK B-related responses insulin receptors associated with glucose uptake in macrophage phagocytosis $F_{c}$ receptors for immunoglobulin $G$, and epidermal growth factor receptors and insulin-like growth factor-2 during proliferation. Compared with healthy controls, increased neuraminidase activity in the plasma of patients with myocardial infarction (MI) has been described [9]. NEU1 is involved in the regulation of various cellular metabolic behaviors and signal transduction. Increased NEU1 expression can promote the expression of inflammatory factors (TNF-a, IL-1, IL-6, etc.). The expression of Neu5Ac, a metabolite downstream of NEU1, was increased in patients with coronary heart disease [10]. However, the role of NEU1 in DCM has not been reported.

Here, the aims of the present study were 1) to determine whether NEU1 is involved in the pathological process of diabetic cardiomyopathy, 2) to identify how does NEU1 affect the development of DCM, and 3) the specific molecular mechanism of NEU1 affecting DCM.

\section{Material and methods}

\section{Materials}

Streptozotocin (STZ) was purchased from Sigma (St Louis, MO, USA). AMPK siRNA and scrambled siRNA were obtained from DesignGene Biotechnology (Shanghai, China). 2, 7-Dichlorofluorescin diacetate (DCFH-DA) was ordered from Nanjing Jiancheng Bioengineering Institute (Nanjing, China). Primary antibodies were purchased from Cell Signaling Technology (Danvers, MA, USA), Abcam
(Cambridge, UK) and Proteintech (Wuhan, China). Secondary antibodies were purchased from LI-COR Biosciences (Lincoln, NE, USA). The cell counting kit-8 (CCK-8) was obtained from Dojindo Laboratories (Kumamoto, Japan). All other chemicals were of analytical grade.

\section{Animals}

C57BL/6J mice were purchased from the institute of Laboratory Animal Science, Chinese Academy of Medical Sciences (Beijing, China). The source of AMPKa global knockout mice has been described previously [11]. Eight to ten-weeks-old (body weight ranging from 20-25g), germ-free male mice were used for the experiments as indicated. All mice were controlled under the condition of $12 \mathrm{~h}$ light/dark cycle at $21^{\circ} \mathrm{C}$ in standard caging and free to diet. All animals' experimental procedures were approved by the Animal Care and Use Committee of Renmin Hospital of Wuhan University.

Animal models constructed on C57BL/6J mice and genetic disrupted AMPK $\%$ mice underpin mechanist evaluation of the diabetic cardiomyopathy. Myocardial injection of adeno-associated virus (AAV9, [ $1 \times 10^{10} \mathrm{vp}$ (viral particles) per animal]) was performed four weeks before STZ injection, and then randomly assigned to STZ injection group or control group. As previously described[12, 13], Diabetic cardiomyopathy model was induced by intraperitoneal (I.P.) injection of streptozotocin (STZ, Sigma, St. Louis, MO) at the dose of $50 \mathrm{mg} / \mathrm{kg}$ dissolved in $100 \mathrm{mM}$ citrate buffer $\mathrm{pH} 4.5$ for 5 consecutive days. Control mice received same volumes of citrate buffer. One week following injections, levels of blood glucose (caudal vein) were measured using a Glucometer (Johnson \& Johnson, USA). Mice with glucose level $>300 \mathrm{mg} / \mathrm{dL}$ were considered as having diabetes and were used for further studies $[12,13]$. AMPK $\%$ mice received the same treatment as described above. Blood samples were collected to evaluate the glucose levels and serum biomarkers. And then mice were euthanized with an overdose of sodium pentobarbital $(200 \mathrm{mg}$ $\mathrm{kg}^{-1}$; i.p.) and the hearts were collected.

\section{Echocardiography and haemodynamic evaluation}

Mice were anesthetized by inhalation of $1.5-2 \%$ isoflurane. Echocardiography was performed to evaluate the structure and function of the left ventricle using a MyLab 30CV system (Biosound Esaote, Inc.) equipped with a $15-\mathrm{MHz}$ probe. To measure the LV end-diastolic dimension (LVEDD), and LV fractional shortening (FS), M-mode tracings derived from the short axis of the left ventricle at the level of the 
papillary muscles were recorded; parameters were obtained from at least three beats and averaged. For the haemodynamic analysis, a 1.4-French catheter-tip micromanometer catheter (SPR-839; Millar Instruments, Houston, TX, USA) was inserted into the left ventricle via the right carotid artery to obtain invasive haemodynamic measurements. An aria pressure volume conductance system (MPVS-300 Signal Conditioner, Millar Instruments, Houston, TX, USA) coupled with a PowerLab/4SP A/D converter was used to continuously record the heart rates, pressure, and volume signals.

\section{Western Blot}

Heart tissues and cultured cardiomyocytes were collected and lysed by RIPA containing phosphatase inhibitors PhosSTOPTM tablets and protease inhibitor cocktail cOmplete mini tablets (Roche). Total protein exacted from the heart tissues and cardiomyocytes was separated by 12\% SDS-PAGE and then were blotted onto PVDF membranes. The membranes would be blocked in 5\% skimmed milk diluted in Tris-buffer saline added Tween-20 (TBST) for $1 \mathrm{~h}$ at room temperature (RT) before incubating with the primary antibodies. The membranes were thoroughly washed in TBST and then incubated with the primary antibodies at $4^{\circ} \mathrm{C}$ overnight. And probed with secondary antibodies for $1 \mathrm{~h}$ at RT after washing the membranes for the second time. The membranes were washed for the last time and incubated with ECL substrate. All the proteins were normalized to GAPDH and the bands were quantified using an image analysis system (Image Lab). The primary antibodies used are listed in Supplementary Table 1.

\section{Real-time PCR}

$1 \mu \mathrm{g}$ total mRNA exacted from the heart tissues and cardiomyocytes using TRIzol regent was worked as the template for the synthesis of cDNA using the Transcriptor First Strand cDNA synthesis kit (Roche). PCR reaction were performed using LightCycler 480 SYBR Green Master Mix and the quantity of the PCR product was normalized to GAPDH. The primers used are presented in Supplementary Table 2.

\section{Determination of insulin content in pancreas}

Briefly, the mice were sacrificed at the specified time point. The pancreatic tissue was carefully removed, and immediately frozen in liquid nitrogen. The samples were homogenized with acidified ethanol (75\% ethanol, $1.5 \% 12 \mathrm{~mol} / \mathrm{L} \mathrm{HCl}$ and $23.5 \%$ $\mathrm{H} 2 \mathrm{O}$ ), incubated at $4^{\circ} \mathrm{C}$ for $72 \mathrm{~h}$, and centrifuged. Then according to the manufacturer's instructions for experimental determination (Beyotime Biotechnology, https://www.beyotime.com/product/PI602 .htm).

\section{Histological analysis, Tunel and Immunohistochemistry staining}

Picrosirius red (PSR), HE staining, Tunel staining and immunohistochemical staining were performed following previously described [13, 14]. Heart tissues collected from all studied groups were fixed with $10 \%$ neutral formalin buffer overnight and embedded with paraffin. The hearts were dissected into $5 \mu \mathrm{m}$ slices. PSR staining was performed to evaluate cardiac fibrosis. HE staining was used to assess the morphological changes of the hearts. All the sections were observed and photographed using a light microscope and a Nikon photoimaging System (Tokyo, Japan) at the magnification of $40 \times$ and $200 \times$. To detect cardiac cell death, terminal deoxynucleotidyl transferase dUTP nick-end labelling (TUNEL) staining was used to detect in situ detection of apoptosis in diabetic hearts, according to the instructions of ApopTag Plus in situ Apoptofluorescein Detection kit (Millipore, MIT, USA). After staining, the slices were observed under the OLYMPUS DX51 fluorescence microscope. The images obtained were blindly analyzed by digital analysis software (Image-Pro Plus 6.0).

For immunohistochemistry, the slices were baked in the oven for over 30min and deparaffined in Xylene 3 times for $15 \mathrm{~min}$. Before hyperthermia antigen retrieval with sodium itrate buffer $(\mathrm{pH} 6.0)$, the slices were hydrated in gradient ethanol (from $100 \%$ to $70 \%$ ). To eliminate the influence of endogenous peroxidases, slices were incubated with $3 \% \mathrm{H}_{2} \mathrm{O}_{2}$ for $10 \mathrm{~min}$ at $\mathrm{RT}$ and then blocked with $8 \%$ goat serum for an hour. Sections were stained with anti-TNFa (Cell Signaling Technology, 11948S), anti-CD45 (ABCAM, ab10558), anti-CD68(ABCAM, ab125212) and anti-cleaved-caspase3 (Cell Signaling Technology, 9664,) at $4^{\circ} \mathrm{C}$ overnight and incubated with EnVisionTM+/HRP reagent at $37^{\circ} \mathrm{C}$ for an hour. To visualize the slices, DAB reagent was used at RT. After that, the slices were mounted with neutral gum and examined using light microscope. For each section, 4-8 visions were counted and a mean value was obtained.

For immunofluorescence staining, paraffinembedded sections of mice heart tissue were sealed with $8 \%$ sheep serum after washing in PBS, and incubated at $4{ }^{\circ} \mathrm{C}$ overnight with mouse anti-aSMA primary antibodies (ABCAM, ab5694). After being washed in PBS, the slices were incubated with the secondary antibody shown at room temperature for 1 hour, and then washed with PBS. The nucleus was stained with 4pyr6-diamino-2-phenylindole (DAPI), Invitrogen, S36939). Fluorescence microscope (OLYMPUS DX51) and DP2-BSW software (version 2.2) were used to obtain images. 


\section{Determination of lipid peroxidation, SOD activity and GSH/GSSG content}

To further assess oxidative stress level, myocardial malondialdehyde (MDA) or 4 hydroxynonenal levels (4-HNE) levels, SOD activities and glutathione (GSH) oxidized glutathione (GSSG) levels in the myocardial tissues were determined using commercially available kits procured from Beyotime Co. (Nantong, China) according to previous study[15].

\section{Cell culture and treatments}

Neonatal rat ventricular myocytes (NRVMs) were separated from newborn rat within 3 days and cultured with Dlbecco's modified Eagle's medium (DMEM) added $10 \%$ fetal bovine serum (FBS) at $37^{\circ} \mathrm{C}$ in incubator gassed with $5 \%$ carbon dioxide. When $60 \%-70 \%$ fusions is reached, NRVMs are exposed to normal glucose (NG, 5.5mmol/1) or high glucose (HG, $33 \mathrm{mmol} / \mathrm{l}$ ) respectively at a specified time point. To investigate the protection of SIRT3 in vitro, NRVMs were transfected with $100 \mathrm{nmol} / 1$ SIRT3 small interfering (siSIRT3) or a control nonspecific siRNA for $4 \mathrm{~h}[16]$. AMPKa knockdown was achieved by siAMPKa transfection using Lipofectamine RNAiMAX (Invitrogen) for $24 \mathrm{~h}$, whose efficiency has been proved in our previous study [14, 17]. To explore the mechanism through shNEU1 activated AMPKa, NRVMs were incubated with STO-609 (the CaMKK inhibitor, $800 \mathrm{nmol} / \mathrm{l}$ ) [18], Takinib (the selective TAK1 inhibitor, $10 \mathrm{mM}$ ) [19]. To knock down LKB1, siLKB1 was used. To induce cell death, NRVMs were subjected to $\mathrm{H}_{2} \mathrm{O}_{2}(200 \mu \mathrm{mol}$ for $12 \mathrm{~h})$ treatment.

\section{Detection of ROS and cell viability}

NRVMs which were seeded in 6-well plates were exposed to HG for 24h. To assess the ROS level, the cells were incubated with DCFH-DA for $0.5 \mathrm{~h}\left(37^{\circ} \mathrm{C}\right)$, and then were visualized in a blinded manner under an Olympus IX53fluorescence microscope. Cell count analysis (CCK-8; Dojindo Molecular Technologies, Rockville, MD, USA) was performed according to the manufacturer's protocol assays for cell viability.

\section{Cardiac ELISA test kits}

The concentrations of NEU1 in cardiac homogenates were measured by enzyme-linked immunosorbent assay (ELISA) methods.

\section{Statistical analysis}

All data were expressed as mean \pm standard error of mean (SEM). Comparisons between two groups were performed using Student's $t$ test. Differences among groups were assessed using a one-way analysis of variance (ANOVA) followed by
Tukey's post hoc test. Probability values of $P<0.05$ were considered significant.

\section{Results}

\section{NEU1 is up-regulated in cardiomyocytes under diabetic conditions}

To investigate whether NEU1 is involved in the pathogenesis of diabetic cardiomyopathy, we first detected NEU1 expression levels by Western blot. The Results showed that NEU1 were significantly increased in the hearts of diabetic mice (Figure 1A). Furthermore, ELISA data confirmed that STZ treatment activated NEU1 expression in mice hearts (Figure 1B). Besides, the elevated NEU1 was mainly localized in cardiomyocytes based on co-localization with Wheat germ agglutinin (WGA) (Figure 1C). We further compared NEU1 expression in cardiomyocytes with or without high glucose. Consistently, the NEU1 protein expressions were markedly increased in the cardiomyocytes confirmed by immunofluorescence staining. Accordingly, time-dependent upregulation of NEU1 was observed in NRVMs treated with high glucose detected by Western Blot (Figure 1E).

\section{Diabetes-induced cardiac dysfunction is rescued by NEU1 inhibition}

To investigate the role of NEU1 in STZ-induced diabetic cardiomyopathy, mice with AAV9 injection were exposed to STZ treatments. As shown in Figure S1A, AAV9-shNEU1\#2 significantly inhibited NEU1 mRNA level in NRVMs comparing with others. The efficiency of AAV9-shNEU1 was presented in Figure S1B. Diabetic mice exhibited increased blood glucose levels (Figure S2A) with a decrease in body weight (Figure S2D). Diabetic mice also had increased glycosylated hemoglobin (HbA1c) levels with a concomitant decline in the pancreas insulin content (Figure S2B, S2C). While, knockdown NEU1 did not significantly alter the body weight/blood glucose level/pancreas insulin content neither in control nor in diabetic animals (Figure S2A-S2D).

Next, we detected the alterations of cardiac function. Consistent with our previous research, mice developed deteriorated cardiac function, as assessed by increased left ventricular end diastolic diameter (LVEDD) and reduced fractional shortening (FS) and LV ejection fraction (LVEF)[13]. NEU1 knockdown rescued the deteriorated cardiac function, as reflected by LVEDD, LVEF and FS (Figure S2E and Figure 2A, $B$ and D). NEU1 inhibition can improve cardiac systolic function (assessed by $\mathrm{dP} / \mathrm{dt} \max$ ) and diastolic function (assessed by $\mathrm{dP} / \mathrm{dt}$ min and E/A ratio) by pressure-volume analysis (Figure 2C and E). 
No significant difference in heart rate among these groups was observed (Figure 2F). However, AAV9-shNEU1 alone showed no beneficial effect on heart function under basal conditions (Figure 2).
Generally, these data indicated that NEU1 inhibition could lead to alleviated cardiac dysfunction in STZ-induced mice.
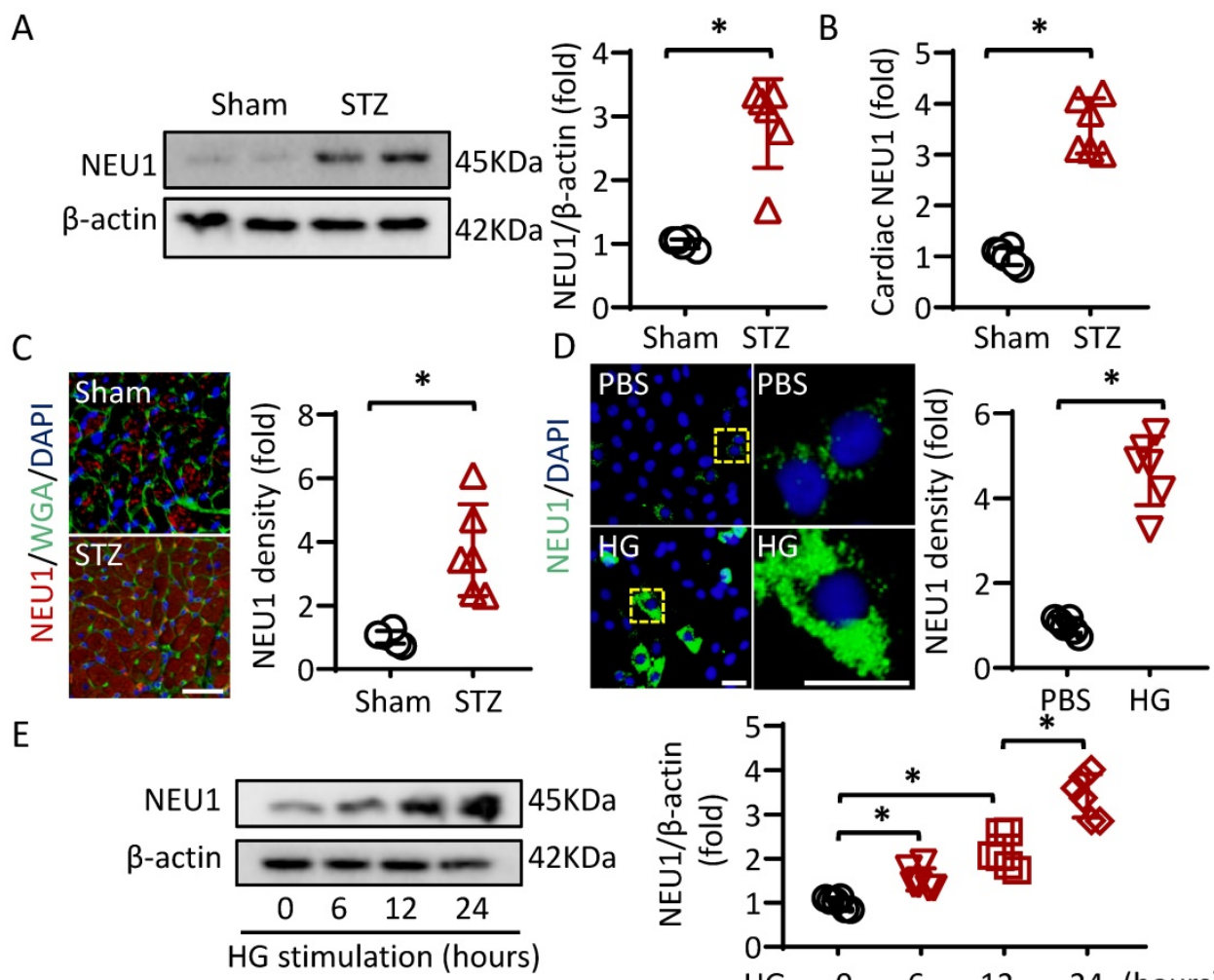

E

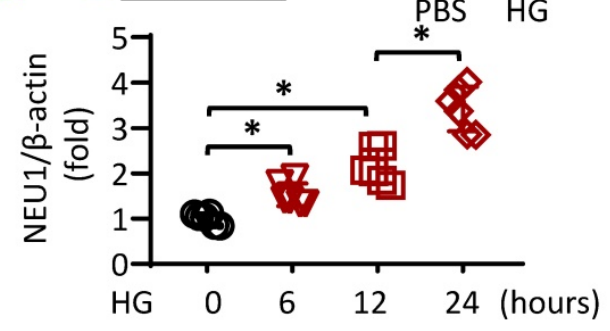

Figure 1. NEU1 is elevated in STZ-induced diabetic mice heart and NRVMs incubated with high glucose. A. Protein levels of NEUI were determined by Western blot in heart samples from Sham and diabetic mice $(n=6)$. B. Cardiac NEUI expression detected by ELISA $(n=6)$. C. Immunofluorescence with an anti-NEU1 antibody in slices from the indicated mice hearts $(n=6)$. scale bar, $50 \mu \mathrm{m}$. D. Immunofluorescence images of NEU1 in NRVMs with or without high glucose $(n=6)$. scale bar, $50 \mu m$. E. Protein levels of NEU1 in NRVMs treated with high glucose for the indicated time $(n=6)$. $* P<0.05$ vs. the matched group.
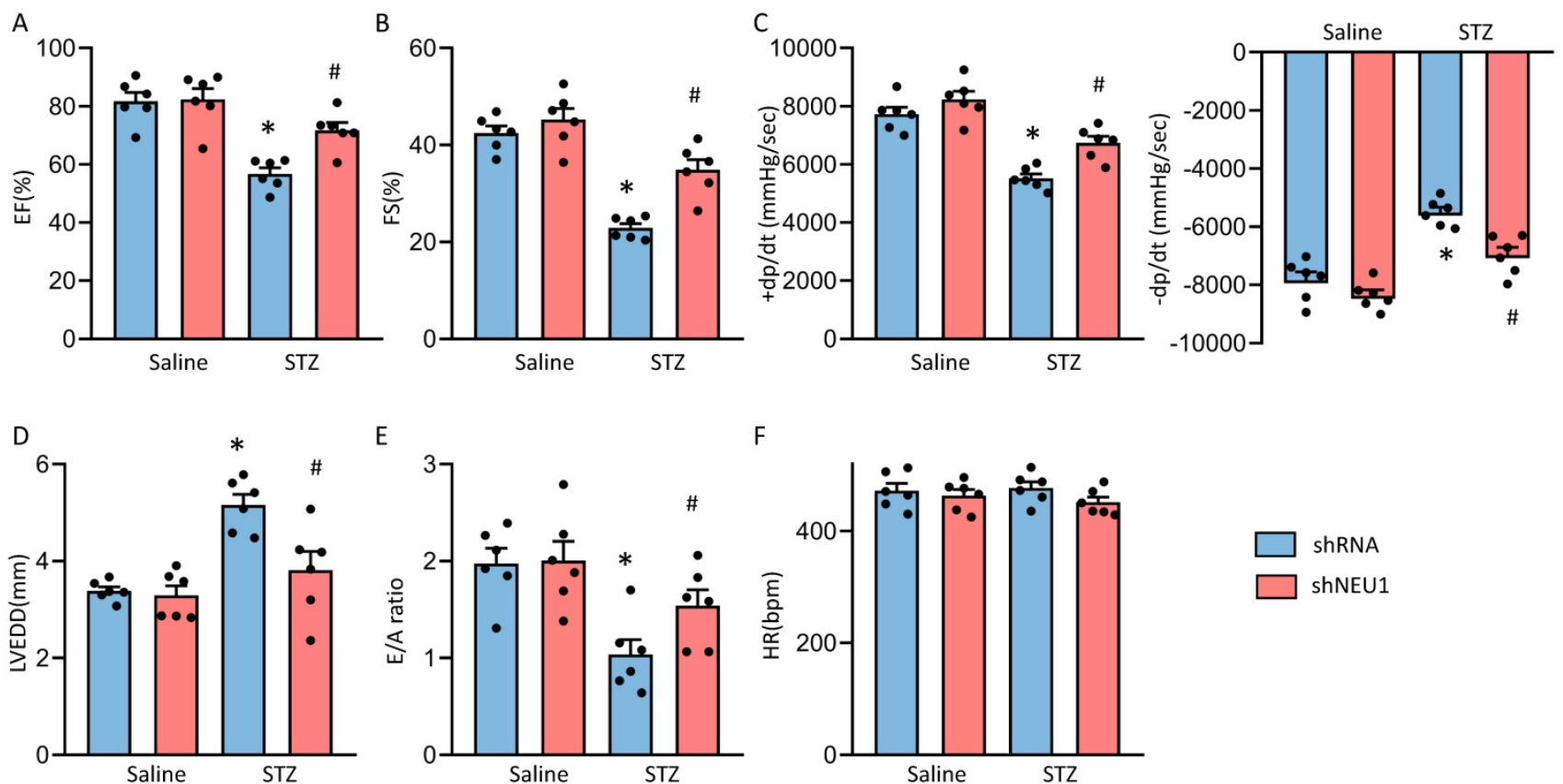

Figure 2. NEU1 inhibition protected against diabetes-induced cardiac dysfunction in mice. A-C. Cardiac function and hemodynamic parameters of mice ( $\mathrm{n}=6$ ). $D$. Left ventricle end-diastolic diameter (LVEDD) $(n=6)$. E. E/A ratio $(n=6)$. F. Heart rate $(n=6) . * P<0.05$ vs. Saline+Vehicle group; $\# P<0.05$ vs. STZ+Vehicle group. 
NEU1 inhibition alleviates cardiac fibrosis in STZ-induced diabetic mice

Next, we determined whether NEU1 inhibition could attenuate fibrosis in STZ-induced diabetic mice hearts. PSR-staining results showed that AAV9-shNEU1 mice presented with less collagen deposition than that in mice with AAV9-shRNA after STZ-induced, which was verified by the suppressed mRNA levels of fibrotic markers, such as connective tissue growth factor $(C T G F)$, transforming growth factor- $\beta$ (TGF- $\beta)$, collagen I ( $\mathrm{Col} I)$, collagen IIII ( $\mathrm{Col}$ III) and fibronectin $(F n)$ (Figure 3A, 3C). Cardiac fibrosis was further verified by immunochemistry of a-smooth muscle actin (a-SMA) (Figure 3A). Also, NEU1 inhibition reduced the expression of a-SMA and Col I in STZ-induced diabetic mice (Figure 3B).

\section{Knockdown of NEU1 suppressed myocardial inflammation accumulation and apoptosis in diabetes heart}

As inflammation and apoptosis are the key features in the pathogenesis of diabetic cardiomyopathy. When upstream signal activates IкBa degradation, NF-кB p65 transfers from inactivated state and from cytoplasm to nucleus, binds to corresponding inflammation-related genes, initiates inflammatory cytokine transcription and induces inflammation [20]. To determine the effect of shNEU1 on inflammation induced by STZ, we examined whether NEU1 inhibition affects inflammatory cell infiltration and NF-kB signaling in the heart. Western blot confirmed that the increase in the phosphorylation level of NF- $\mathrm{kB}$ family member p65 caused by STZ were inhibited by NEU1 inhibition (Figure 4A,4B). IKBa phosphorylation and IкBa degradation in vehicle-treated diabetic mice was significantly impaired in diabetic mice with NEU1 inhibition (Figure 4A,4B). Also, NEU1 knockdown can decreased the inflammatory response in STZ-induced diabetic mice hearts, as manifested by the decreased mRNA level of $I L-6, T N F-a$ and $M C P-1$ compared with the vehicle-treated diabetic group (Figure 4C). Furthermore, the infiltration of CD68-labeled macrophages and CD45-labeled leukocytes in diabetic mice increased significantly. In contrast, the migration and accumulation of inflammatory cells was alleviated by NEU1 inhibition (Figure 4D,4E). The results of TUNEL staining and immunohistochemical of cleaved caspase-3 revealed that NEU1 knockdown can inhibit apoptosis induced by STZ injection (Figure 4F, 4G). The protective effect on apoptosis was further confirmed by Western blot showing that NEU1 inhibition limited the alterations of the levels of BAX, C-caspase3 and BCL-2 level in diabetic hearts (Figure 4H).
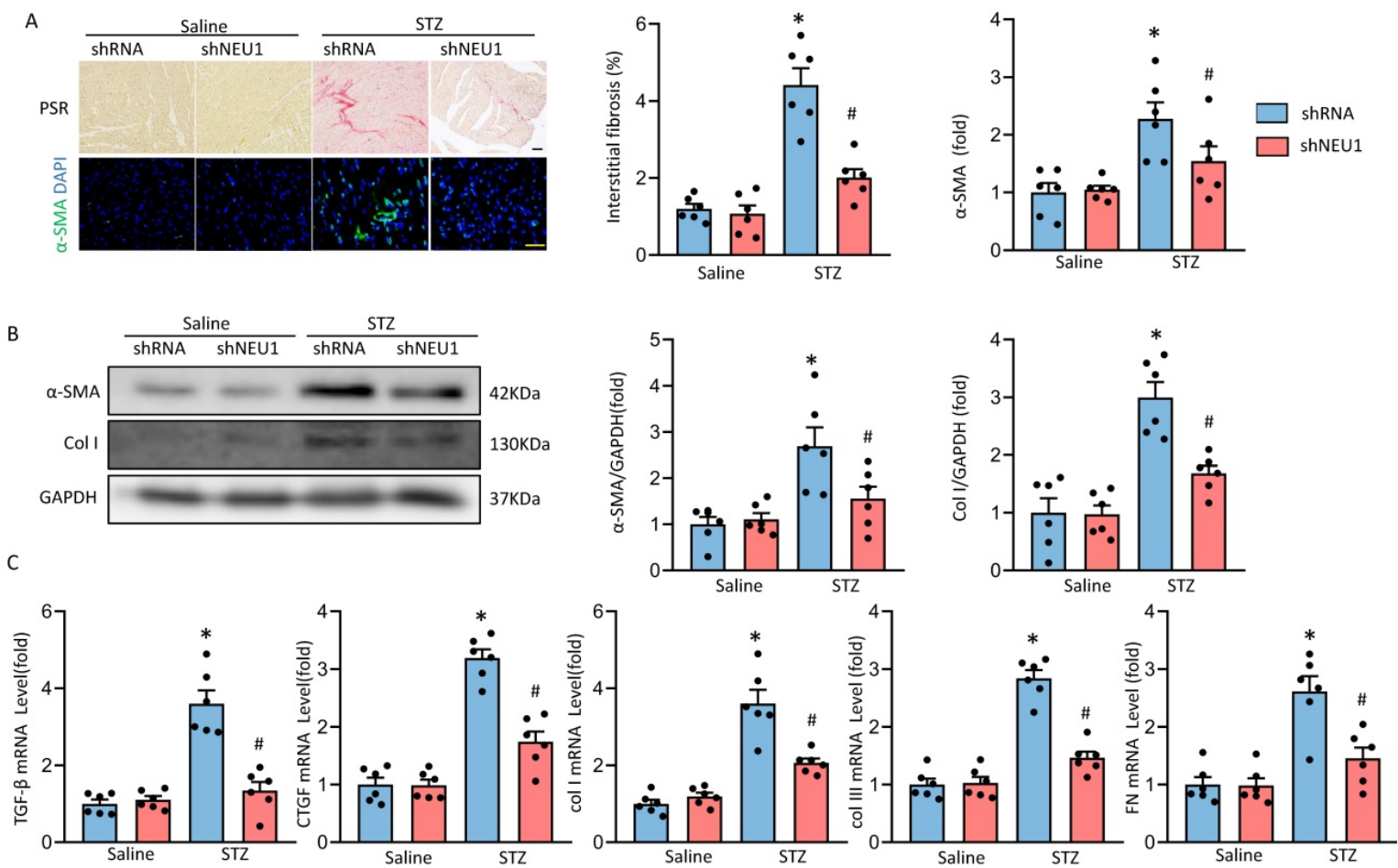

Figure 3. NEU1 inhibition attenuates diabetes-associated myocardial fibrosis. A. Representative images of the morphological analysis of cardiac fibrosis as reflected by PSR staining and immunofluorescence staining for a-SMA (bar $=50 \mu \mathrm{m})$. Quantification of fibrotic areas $(n=6)$. Quantitative results of a-SMA of immunofluorescence staining showing the activation of myofibroblasts $(n=6)$. B. $\alpha$-SMA and collagen I (col I) protein expression and quantitative data $(n=6)$. C. The relative mRNA levels of TGF- $\beta$, connective tissue growth factor (CTGF), collagen I (col I), collagen III (col III), and fibronectin (FN) normalized to GAPDH in mice $(n=6)$. $* P<0.05$ vs. Saline $+V$ ehicle group; $\# P<0.05$ vs. STZ+Vehicle group. 


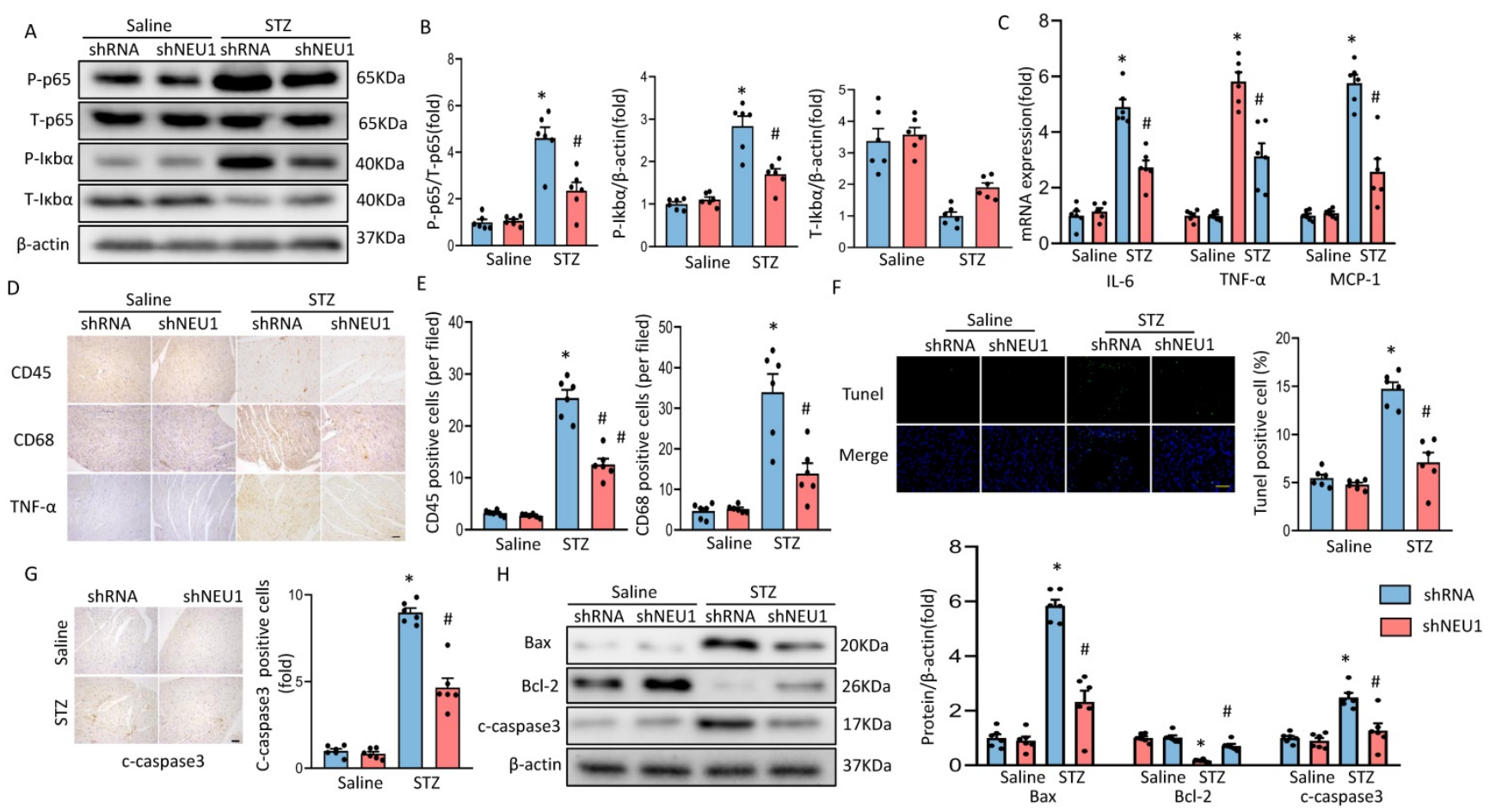

Figure 4. NEU1 inhibition suppressed myocardial inflammation accumulation and apoptosis in diabetes heart. A-B. Total and phosphorylated $\mathrm{p} 65$ and IKBa protein expression and quantitative data $(n=6)$. C. The relative mRNA levels of IL-6, TNF- $\alpha$, and MCP-1 normalized to GAPDH in mice ( $n=6)$. D-E. Representative images of immunohistochemistry analysis of CD45, CD68, and TNFa in diabetic hearts. Quantitative data $(n=6)$. F. Myocardial apoptosis measured by TUNEL staining in heart sections $(n=6,10$ fields per coverslip, bar $=50 \mu \mathrm{m})$. G. Cleaved caspase- 3 expression in the heart sections of the saline and diabetic mice determined by immunohistochemistry $(n=6,10$ fields per coverslip, bar $=50 \mu \mathrm{m}$ ). $\mathrm{H}$. Western blot and quantitative analysis showing the protein levels of Bax, Bcl-2 and cleaved caspase-3 in vehicle and shNEUI treated mice $(\mathrm{n}=6) .{ }^{*} P<0.05$ vs. Saline+Vehicle group; $\# P<0.05$ vs. STZ+Vehicle group.

A

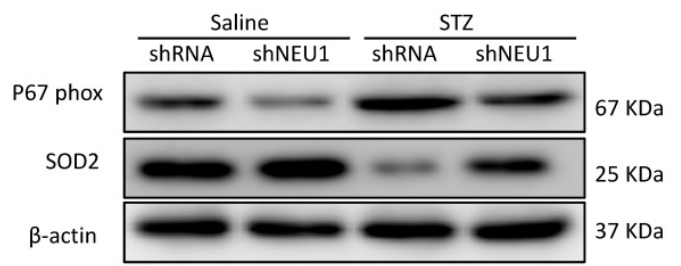

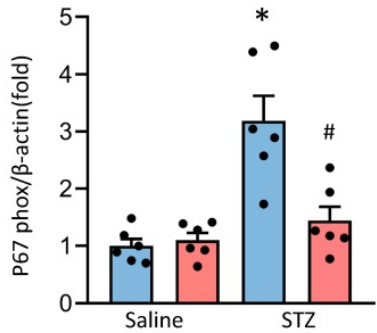
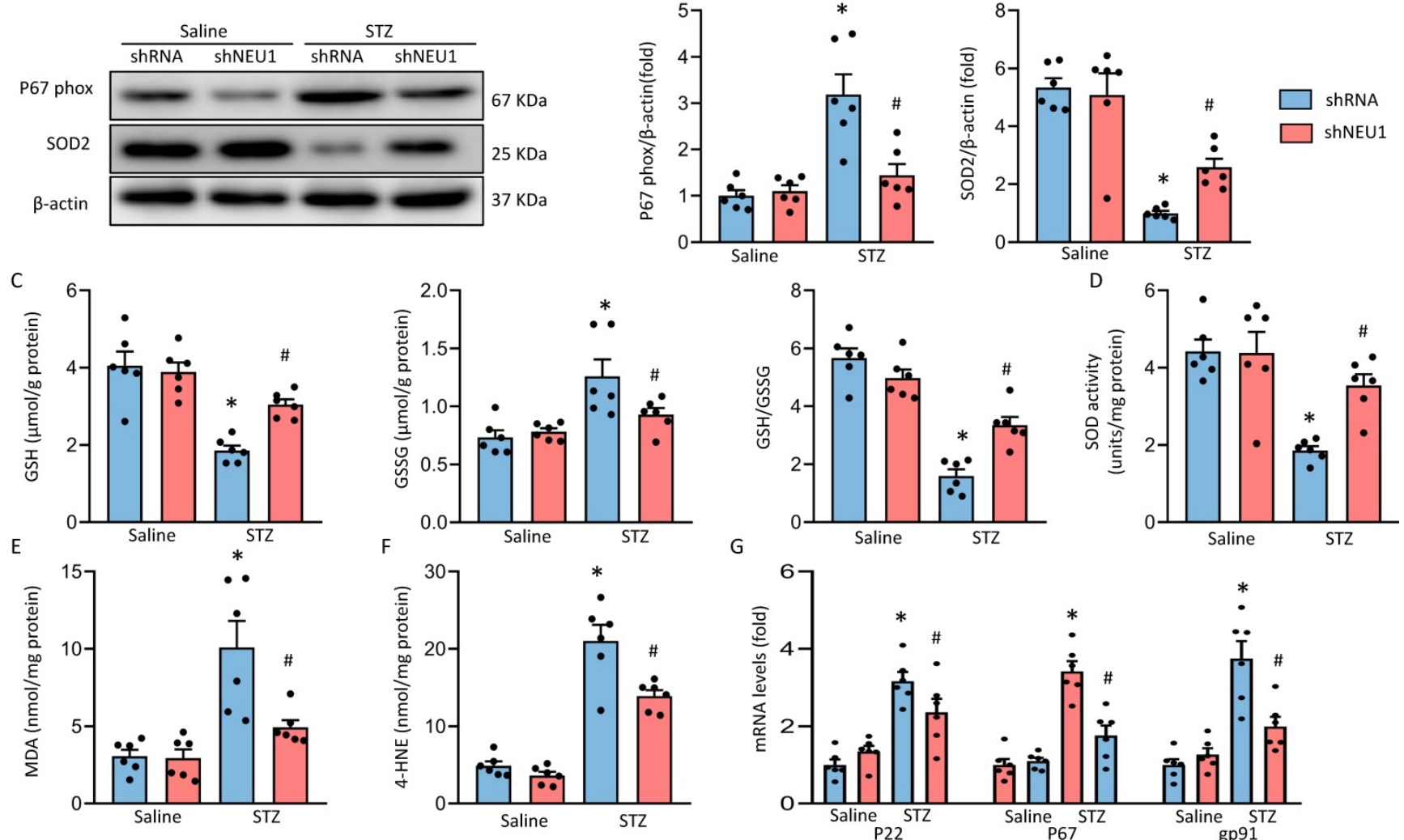

Figure 5. shNEU1 attenuated diabetes-induced oxidative stress in the hearts. A-B. Western blot and quantitative analysis showing the protein levels of p67 phox and SOD2 in vehicle and shNEU1 treated mice $(n=6)$. C. Endogenous antioxidants $(G S H)$ content $(n=6)$. D. Total SOD activity in diabetic mice $(n=6)$. E. Lipid peroxidation in diabetic hearts $(n=6)$. F. 4-HNE $(n=6)$. G. NADPH oxidase subunits mRNA expression by real time RT-PCR $(n=6)$. *P<0.05 vs. Saline+Vehicle group; $\# P<0.05$ vs. STZ+Vehicle group. 


\section{NEU1 inhibition attenuates diabetes-induced myocardial oxidative stress}

As oxidative stress is the main feature of diabetic cardiomyopathy. Western blot results verified that NEU1 inhibition significantly decreased the P67phox and increased SOD2 expression in STZ-induced diabetic mice (Figure 5A, 5B). There was increased accumulation of GSSG with concordant decrease of GSH and GSH/GSSG ratio in heart of diabetic mice. While, these alternations were attenuated when mice treated with AAV9-shNEU1 in response to STZ injection (Figure 5C). Compared with mice in the control groups, total SOD activity was significantly reduced in diabetic mice and was increased by administration of AAV9-shNEU1 (Figure 5D). As shown in Figure 5E and 5F, NEU1 inhibition decreased lipid peroxidation in diabetic mice. Also, our results confirmed that the upregulation of P67phox, P22phox and gp91phox mRNA induced by STZ injection was reduced in diabetic mice with AAV9-shNEU1 administration (Figure 5G). Collectively, these data indicated that NEU1 inhibition exerted antioxidation effects in diabetic hearts.

\section{NEU1 promotes HG-induced cardiomyocyte injury by inhibiting SIRT3}

It is reported that Sirtuin-3 (SIRT3) plays an important role in mitochondrial homeostasis and plays a protective role in the occurrence and development of DCM [21, 22]. Consistent with previous studies[23], we observed that STZ significantly inhibited the expression of SIRT3 at the level of mRNA and protein. Whereas, the inhibitory effect of STZ was counteracted by AAV9-shNEU1 administration (Figure 6A and Figure S3A.). Given the observation that NEU1 inhibition activated SIRT3 in vivo, we examined the role of SIRT3 in NEU1-mediated adverse effects in vitro. NRVMs were infected with siRNA knock down SIRT3 and the efficiency was confirmed by Western blot (Figure S3B). Accumulating evidences have shown that SIRT3 exerted cardiovascular protection roles by regulating oxidative stress [24]. Intriguingly, administration of shNEU1 induced a significant reduction in ROS, a substantial decrease in mRNA of inflammatory mediators, and a significant increase in cell viability in NRVMs exposed to HG 24h. Consistent with the result in vivo, we found that the inhibitory effect of shNEU1 on ROS generation was blunted after the knock-down of SIRT3 (Figure 6B,6C and Figure S3C). Besides, NEU1 inhibition lost its effect on HG-induced inflammatory response in SIRT3-knockdown cells, as shown by the unaltered mRNA levels of IL-6, TNF-a and MCP-1 (Figure 6D). NRVMs exposed to HG for $72 \mathrm{~h}$ had decreased cell viability, but the cell viability was increased when administration with shNEU1 (Figure 6E). HG also downregulated BCL-2 and upregulated BAX in NRVMs. However, these alternations are blocked by NEU1 inhibition (Figure $6 \mathrm{E}$, Figure S3D). These effects of shNEU1 have been abolished by SIRT3 deficiency. Furthermore, $\mathrm{H}_{2} \mathrm{O}_{2}$ were used to induce myocyte apoptosis. SIRT3 knockdown completely abolished the protection by NEU1 inhibition against $\mathrm{H}_{2} \mathrm{O}_{2}$-induced apoptosis (Figure S3E). Consistent with the changes of molecules, we found that SIRT3 knock-down negated beneficial effects mediated by NEU1 inhibition on lipid peroxidation and oxidative damage in HG-treated NRVMs, as confirmed by the unaltered MDA, and SOD activity (Figure S3F). These results indicated that NEU1 promotes HG or STZ-induced oxidative stress, inflammatory response and cardiomyocytes apoptosis via downregulating the expression of SIRT3.

\section{AMPKa accounts for the key role of the SIRT3/SOD2 pathway in vitro}

Next, we discussed the possible mechanism of NEU1 promoting STZ-induced downregulation of SIRT3. Previous studied have found that the phosphorylation of AMPKa was decreased in diabetic cardiomyopathy. AMPK alleviates cardiac remodeling parameters and improves cardiac function through SIRT3/oxidative stress signal pathway [25]. Hence, we supposed that NEU1 inhibition might prevent STZ-induced downregulation of SIRT3 via activating AMPKa. As expected, we found that shNEU1 treatment restored the reduction of AMPKa phosphorylation induced by HG in NRVMs (Figure 7A).To further prove the hypothesis that AMPKa was responsible for the upregulation of SIRT3, we knocked down AMPKa with siRNA, and the efficiency was evidenced by western blot (Figure S4A).Western blot results showed that AMPKa knockdown abrogated the restoration of SIRT3 and SOD2 level by NEU1 inhibition in vitro (Figure 7B). Consistent with the downregulation of SIRT3,we found that AMPKa knockdown significantly blocked the inhibitory effect of NEU1 inhibition on ROS accumulation and oxidative stress level (Figure S4B-S4C).In addition, the protective effect of NEU1 inhibition on inflammatory response and cell death was also blunted, as evaluated by the mRNA level of IL-6, TNF- $\alpha$ and cell viability (Figure S4D-S4F). Hence, we suggested that AMPKa accounts for the key role of SIRT3/SOD2 pathway. 

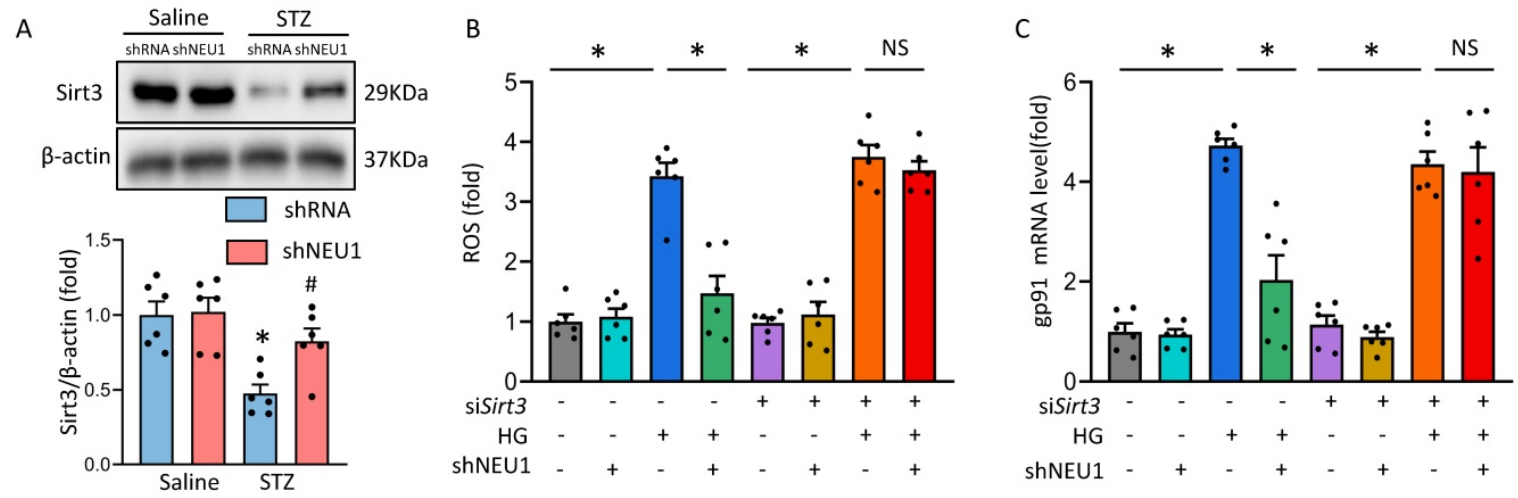

D
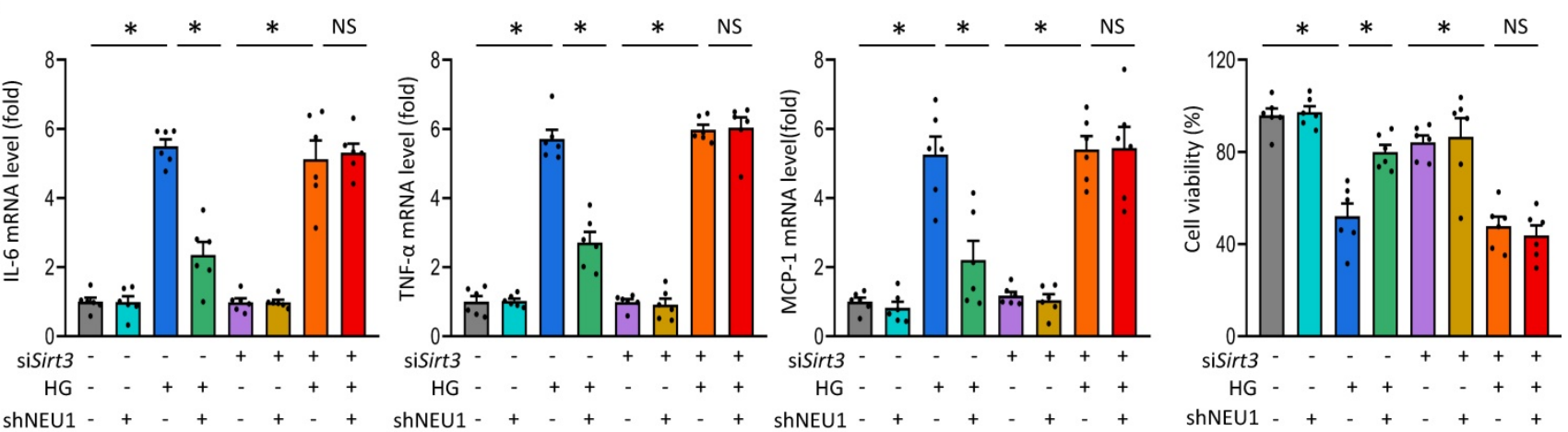

Figure 6. NEU1 promotes HG-induced adverse changes via suppressing SIRT3 in vitro. A. Western blot and quantitative analysis showing the protein levels of SIRT3 in vehicle and shNEU1 treated mice $(n=6)$. $* P<0.05$ vs. Saline+Vehicle group; $\# P<0.05$ vs. STZ+Vehicle group. B. SIRT3 knockdown offset the protection afforded by NEU1 inhibition against ROS induced by HG $(24 h)(n=6)$. C. The relative mRNA levels of gP9l normalized to GAPDH under HG stimulation NRVMs $(n=6)$. D. The relative mRNA levels of IL-6, TNF- $\alpha$, and MCP-1 normalized to GAPDH under HG stimulation in NRVMs $(n=6)$. E. Cell death was detected by an CCK8 assay under HG stimulation in NRVMs $(n=6) . * P<0.05$ vs. the matched group; NS indicates not significant.

A

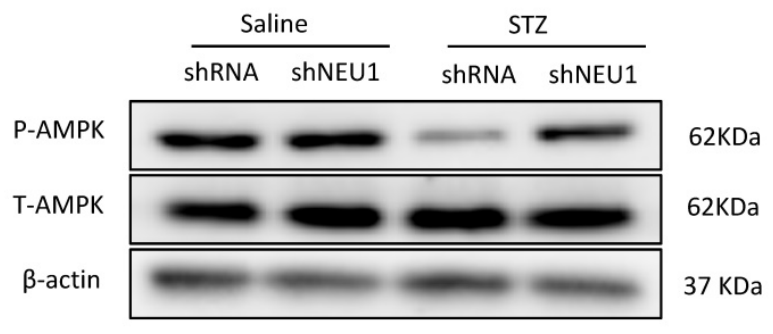

B

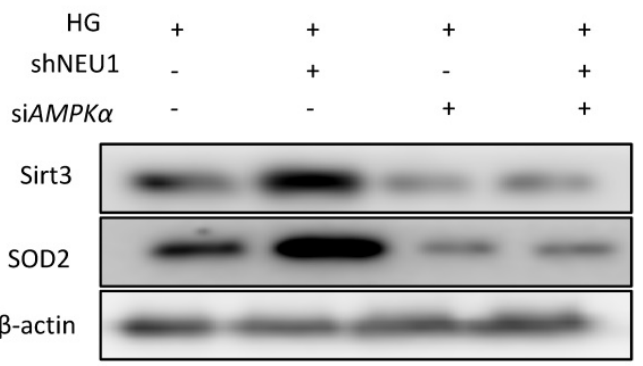

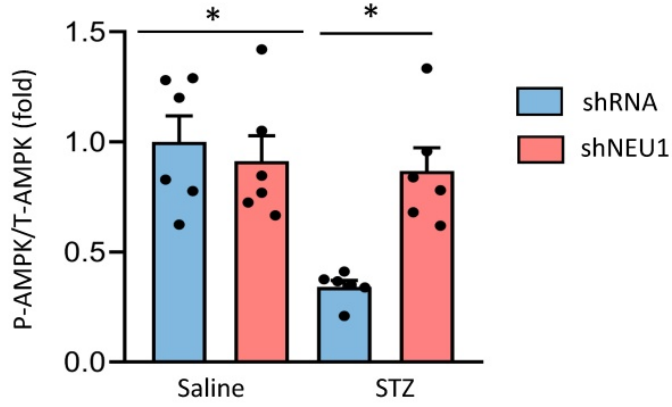

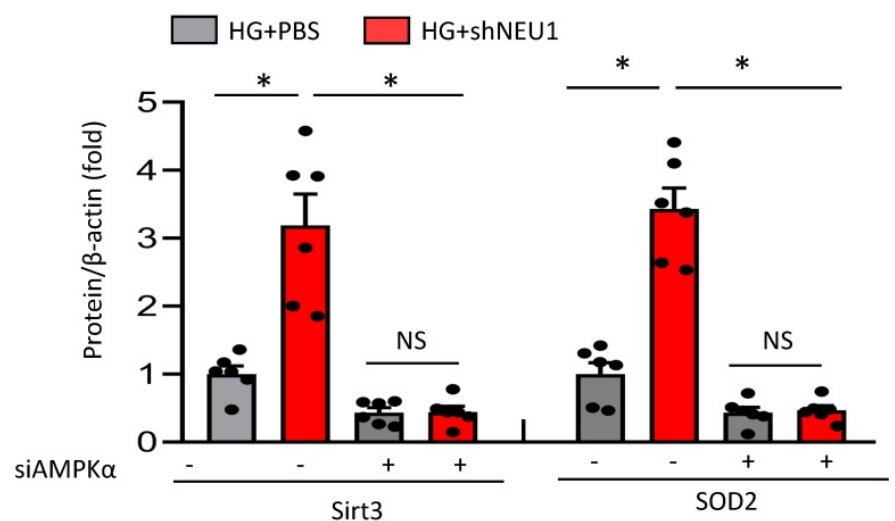

Figure 7. AMPKa accounts for the key role of the SIRT3/SOD2 pathway. A. Total and phosphorylated AMPKa protein expression and quantitative data ( $=6$ ). $\# P<0.05$ vs. STZ+Vehicle group. B. SIRT3 and SOD2 levels in NRVMs transfected with or without siAMPKa under HG stimulation ( $\mathrm{n}=6$ ). $* P<0.05$ vs. the matched group; NS indicates not significant. 


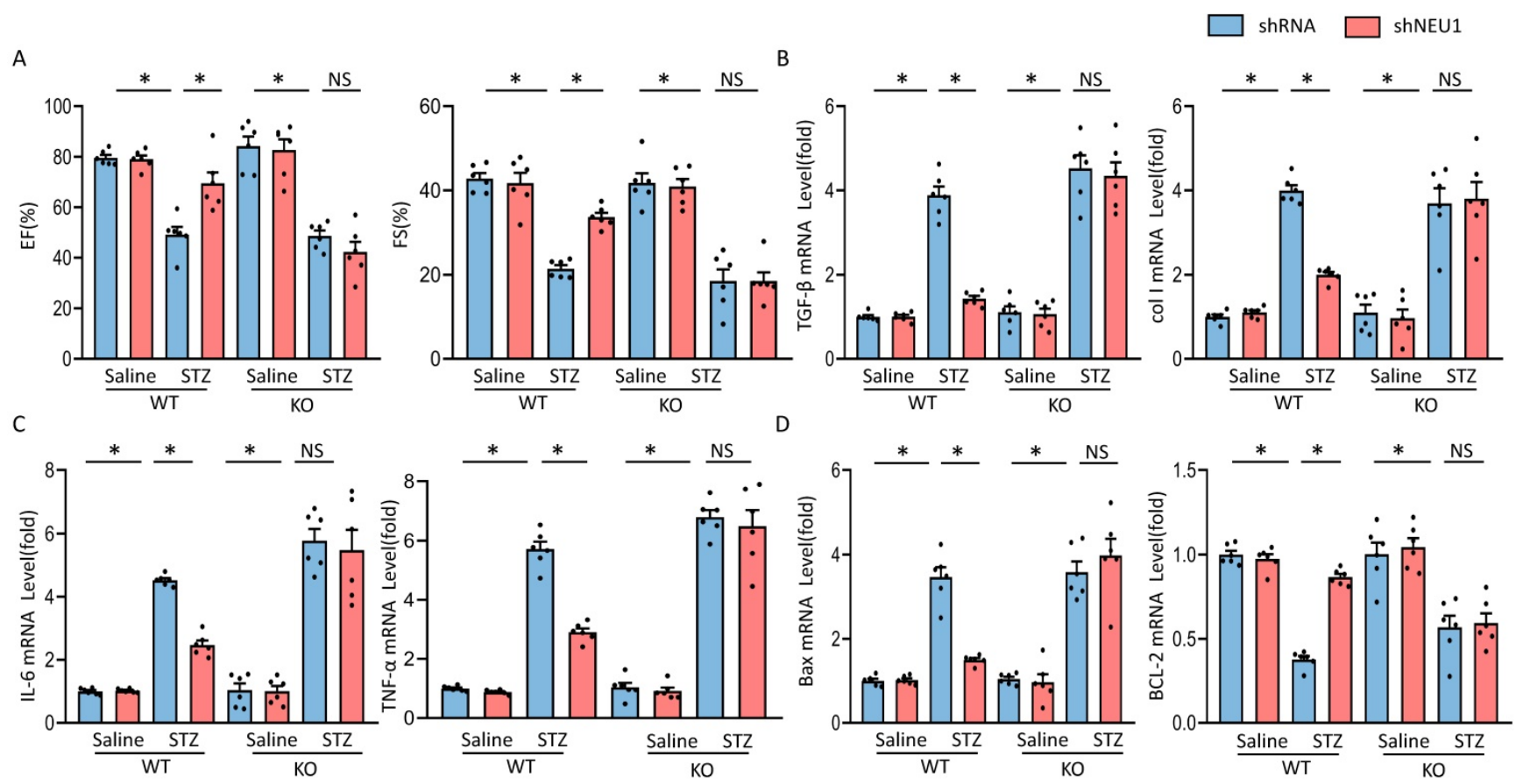

Figure 8. AMPKa deficiency offset the protective effects of shNEU1 in vivo. A. Heart function evaluated by echocardiography ( $\mathrm{n}=6$ ). B. mRNA levels of the fibrotic markers $(n=6)$. C. mRNA levels of the inflammatory markers $(n=6)$. D. mRNA levels of the apoptosis-related genes $(n=6)$. $* p<0.05$ vs. the matched group; NS indicates not significant.

\section{AMPKa deficiency offset the protective effects of shNEU1 in vivo}

To further investigate whether the protective effects of NEU1 inhibition via AMPKa in vivo, AMPKa knockout $(\mathrm{KO})$ mice were used. In line with the data in vitro, we observed that NEU1 inhibition lost the protective effect in AMPKa knockout mice, as evidenced by the indistinguishable LVEF and LVFS (Figure 8A). Besides, shNEU1-mediated downregulation of TGF- $\beta$ and col I was all reversed by AMPKa deletion (Figure 8B). Consistent with the findings in vitro, shNEU1 lost its protective effects on inflammatory response in AMPKa-deficient mice, as reflected by the mRNA level of TNF- $a$ and IL- 6 (Figure $8 \mathrm{C})$. Furthermore, AMPKa KO abrogated the protective effects of NEU1 inhibition on cardiomyocytes apoptosis in response to STZ injection, as indicated by mRNA level of $B A X$ and $B C L-2$ (Figure 8D). Collectively, these data indicated that the protective of shNEU1 were dependent upon the activation of AMPKa.

\section{The precise mechanism of NEU1 regulating AMPKa}

At present, it has been found that there are three upstream kinases of AMPK, namely liver kinase B1(LKB1), transforming growth-factor- $\beta$-activated kinase-1(TAK1), $\mathrm{Ca}^{2+} /$ calmodulin-dependent protein kinase kinase- $\beta(\mathrm{CaMKK} \beta)$ respectively [26]. We investigated the involvement of AMPK in the
shNEU1-triggered activation of SIRT3 signaling. As shown in the previous experimental results, shNEU1 administration improved the phosphorylation level of the AMPKa and the expression level of SIRT3 and SOD2. Next, Takinib (a potent and selective TAK1 inhibitor), STO-609 (a selective CaMKK $\beta$ inhibitor) and small interfering RNA of LKB1(siLKB1) were used. As shown in Figure 9A and 9B, knockdown of LKB1 prevented shNEU1-triggered AMPKa activation. However, Takinib and STO-609 had no such effect on the activation of AMPKa (Figure 9A,9B). Corresponding to this change, after knocking down LKB1, the expression levels of SIRT3 and SOD2 did not increase after shNEU1 administration. Moreover, Takinb and STO-609 did not inhibit the expression of SIRT3 and SOD2 (Figure 9A ,9B). In addition, the protection effects of NEU1 inhibition against HG-induced cell loss was also interdicted by SiLKB1, but not Takinb and STO-609 (Figure 9C). Collectively, these results suggested that NEU1 might inhibits AMPKa via the suppression of LKB1.

\section{Discussion}

In the present study, we demonstrated that NEU1 inhibition alleviated deteriorated cardiac function in diabetic hearts, and protected against diabetes-related cardiac fibrosis, inflammation, oxidative damage and cell death in vivo. In this study, NEU1 inhibition also attenuated HG-induced production of ROS and inflammation, and reduced the cell loss induced by HG in vitro. Mechanistically, 
NEU1 inhibited AMPKa, and thus suppressed SIRT3 to promote subsequent oxidative damage, inflammatory response and cell lose in respond to the high glucose. We also found that NEU1 inhibition activated AMPKa via LKB1. Our data suggested that NEU1 inhibition exert its protective effect against diabetic cardiomyopathy via LKB1-AMPKa-SIRT3 pathway (Figure 10).
A

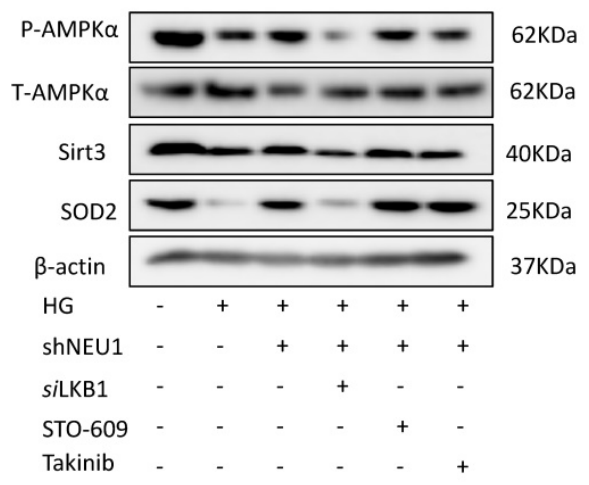

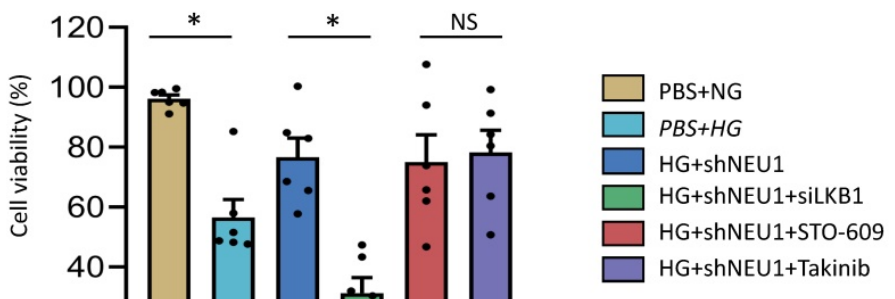
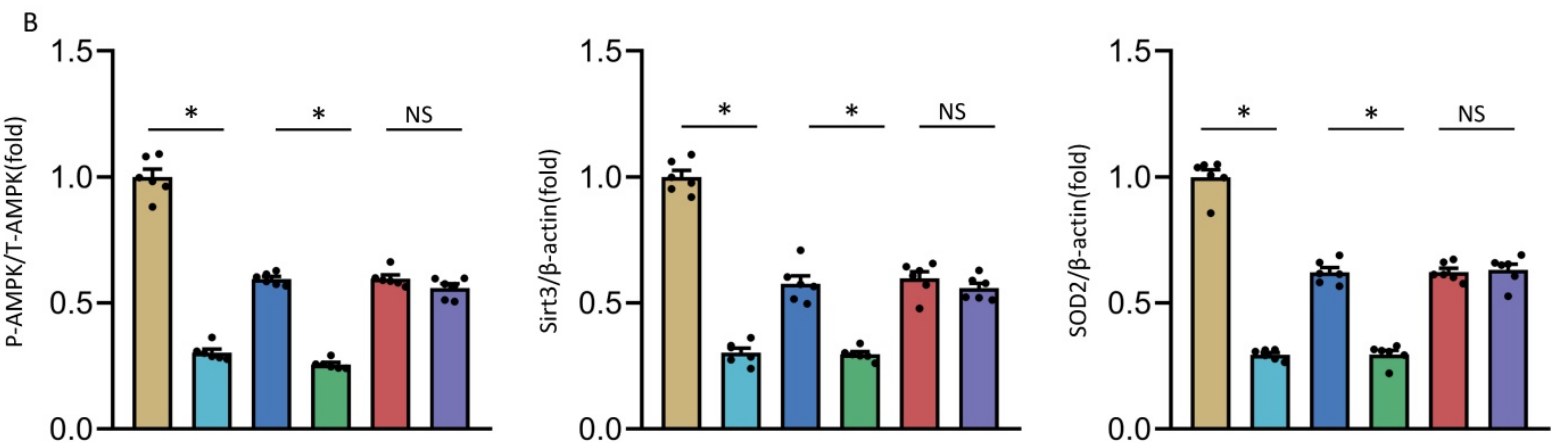

Figure 9. The precise mechanism by which shNEUI activates AMPKa. A-B. p-AMPKa, SIRT3 and SOD2 levels in NRVMs treated with siLKBI, STO-609 (the CaMKK inhibitor, $800 \mathrm{nmol} / \mathrm{l})$, Takinib (the selective TAK1 inhibitor, $20 \mathrm{mmol} / \mathrm{ml}$ ). C. Cell viability of NRVMs $(n=6) * P<0.05$ vs. the matched group; NS indicates not significant.

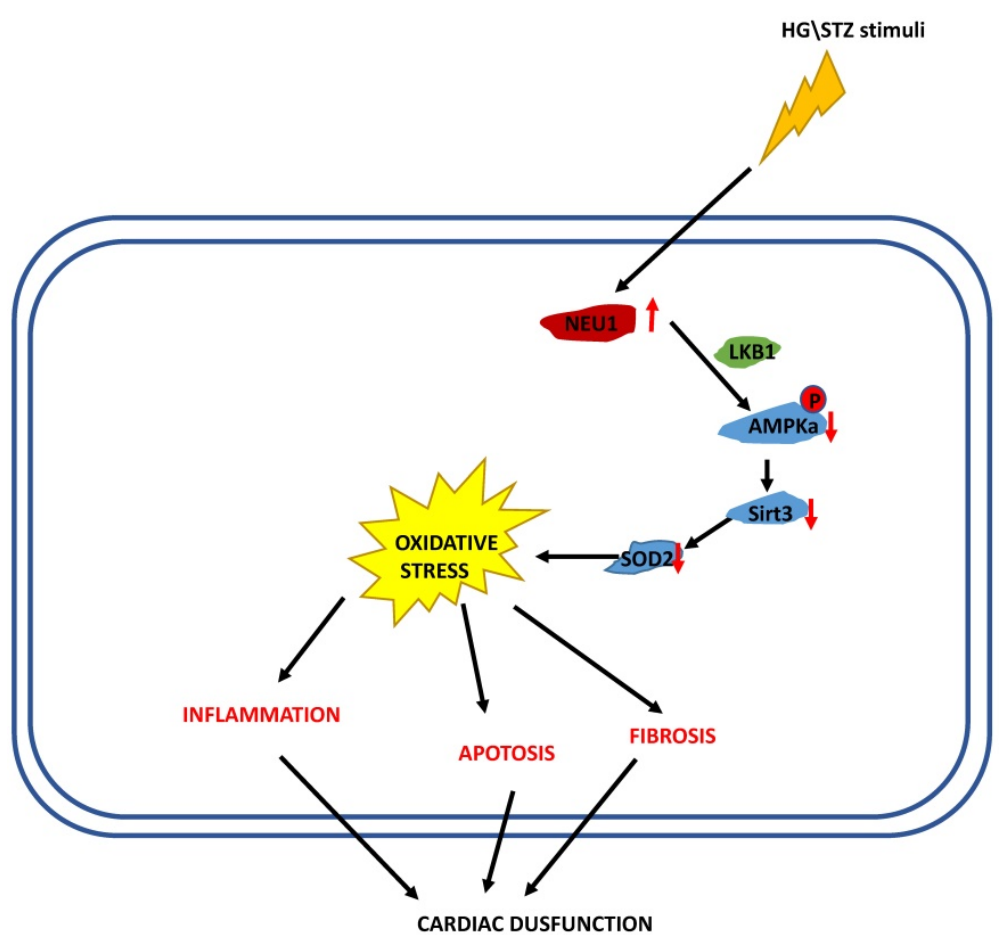

Figure 10. The proposed mechanism of NEUI promotes diabetic cardiomyopathy 
NEU1 is highly expressed in different types of immune cells, such as macrophages in atherosclerotic arteries, circulating monocytes and invading monocytes/macrophages in the heart [27, 28]. It has been reported that NEU1 is involved in the development of inflammatory response and is involved in atherosclerosis and heart failure [29]. In the mononuclear blood cells of patients with myocardial infarction, the expression of NEU1 increased compared with healthy controls. During carotid atherosclerosis, NEU1 is highly expressed in macrophages located in the intimal layer, calcified area, and outer membrane of the plaque [27]. Isoproterenol can rapidly increase the sialidase activity of rat cardiomyocytes and cardiomyocytederived H9c2 cells [30]. Recent studies have found that silencing or pharmacological inhibition of NEU1 prevented cardiac remodeling in response to pressure overload, inhibited cardiomyocytes hypertrophy and attenuated ischemia-related cardiac injury [10, 31]. Despite their shared the cardioprotective effects of NEU1 deficiency in different cardiovascular diseases, our study contributes to supplement of the specific mechanism of NEU1 in the pathogenesis of diabetic cardiomyopathy. This work found that cardiomyocyte-localized NEU1 was increased significantly in STZ-induced diabetic mice heart and HG-stimulated cardiomyocytes. It is true that lowering blood glucose and increasing insulin sensitivity could improve the prognosis of diabetic cardiomyopathy. However, the aim of our experiment is mainly to explore the role of heart NEU1 in the process of diabetic cardiomyopathy. Therefore, we used myocardial injection of AAV9 to inhibit the expression of NEU1 in the myocardium. Moreover, AAV9-derived vectors displaying selective loss of liver tropism and demonstrating potential for cardiac and musculoskeletal gene transfer applications [32]. Therefore, cardiac specific silencing NEU1 does not affect insulin sensitivity or hyperglycemia. This study combined with previous work suggests that NEU1 is a key driver of cardiovascular disease and is therefore a potential therapeutic target.

Studies have shown that the mitochondrial Sirtuin family is involved in insulin resistance in patients with diabetes [23, 33-35]. SIRT3 can prevent or even reverse retina, bone and heart damage caused by diabetes. SIRT3-FOXO3A-Parkin signal pathway may play an important role in the occurrence and development of diabetic cardiomyopathy [36]. Melatonin protects diabetic cardiomyopathy through MST1/SIRT3 signaling pathway [37], while garlic protects diabetic cardiomyopathy from oxidative stress by enhancing SIRT3 activity [38]. In addition, APLN gene therapy can promote angiogenesis and improve the recovery of cardiac function in diabetic cardiomyopathy by up-regulating SIRT3 pathway [39]. Obese mice fed with high-fat diet (HFD) had lower levels of SIRT3 in the heart, while mice with SIRT3 knockout had higher cardiac adipotoxicity [23, 33, 34]. SIRT3 may regulate adipotoxicity by promoting lipid metabolism, reducing fatty acid accumulation and oxidative stress injury [40-42], and restoring cardiac remodeling function [33, 34]. Based on the above research achievements, we detected SIRT3 expression in mice heart injected with STZ. As expected, the expression of SIRT3 decreased significantly in vivo. However, shNEU1 inhibited SIRT3 inactivation and significantly attenuated cardiac dysfunction and cardiac remodeling. In addition, knockdown of SIRT3 abrogated the beneficial effects in vitro. SIRT3 protects pancreatic cells from adipotoxicity by antagonizing cell damage induced by oxidative stress [43]. With the increase of SIRT3 expression, SOD2 deacetylation decreases and SOD2 activity increases, this limits the accumulation of ROS [44]. While it is true that SIRT3 depletion was linked to hyperacetylation of the key mitochondrial antioxidant, SOD2, leading to SOD2 inactivation and mitochondrial oxidative stress [45, 46]. As prior study reported, SIRT3 directly deacetylates and activates mitochondrial isocitrate dehydrogenase 2 (Idh2), leading to increased NADPH levels and an increased ratio of reduced-to-oxidized glutathione in mitochondria [47]. The elevated NADPH, in turn, is necessary for glutathione reductase, which converts GSSG into reduced glutathione GSH, the cofactor used by mitochondrial glutathione peroxidase (GPX) to detoxify ROS. Interestingly, another target of SIRT3, glutamate dehydrogenase (GDH). Moreover, mitochondrial ROS positively regulates NADPH oxidase subunits expression and activation under pathological conditions [48, 49]. Therefore, SIRT3 regulate the expression of $\mathrm{NAPDH}$ oxidase via mitochondrial ROS.

Oxidative stress can induce abnormalities of calcium handling, which subsequently lead to diabetic cardiomyopathy and cardiac dysfunction [50]. NEU1 plays an important role in cellular oxidative stress. Nrf2 activates the mechanical sensitivity of the endogenous antioxidant system is the key for ECs to adapt to oxidative stress in high shear stress areas. The down-regulation of endogenous sialidase NEU1 leads to imbalance of sialic acid and increased expression of Nrf2 target genes, directly involved in the regulation of Nrf2 signaling pathway by sialic acid through unidirectional shear stress. This leads to abnormal EC phenotypes and even atherosclerosis [51]. Increases in ROS and inflammation promote interstitial collagen 
deposition, which is associated with interstitial fibrosis and impaired myocardial relaxation. Increasing the activity of $a-S M A, T G F-\beta$ and functional decreased active matrix metalloproteinase-2 $(M M P-2)$ play an important role in the development of cardiac fibrosis. Structural abnormalities associated with diabetic cardiomyopathy include cardiomyocyte necrosis, progressive abolition of muscular fibrils, collagen formation in connective tissue, and fibrosis [52]. The data in our study suggested that NEU1 inhibition protected against diabetes-induced oxidative damage in vivo and in vitro.

In addition, we also found that high glucose-induced inflammation respond and apoptosis were prevented by shNEU1 administration, but promoted by SIRT3 deficiency in vitro. In addition to regulating oxidative stress, upregulation of NEU1 can promote the inflammatory invasion of monocytes/macrophages, enhance cardiomyocyte hypertrophy, weaken the function of gap junctions, and lead to heart failure. Increased NEU1 expression in cells can promote the expression of inflammatory factors (TNF-a, IL-1, IL-6, etc.). NEU1 has been reported to be involved in the expression of TLRs and the regulation of downstream signaling pathways [53, 54]. In human T cells, both NEU1 and NEU3 mRNA can be significantly induced by $\mathrm{T}$ cell receptor stimulation, and some cytokines, including interleukin (IL)-2 and IL-13, can be induced by the up-regulation of these salivary enzymes [55]. These anti-inflammatory and anti-oxidative actions might explain the cardioprotection of shNEU1 on diabetic mice. Cardiac cell loss is one of the main factors that resulted in the impairment of cardiac function. Here, we also found that cells apoptosis in diabetic hearts was attenuated by NEU1 inhibition but aggravated by SIRT3 deficiency. Here we found that shNEU1 increased SIRT3 expression in vivo and in vitro. Our studies suggest that the elevated of SIRT3 following NEU1 inhibition is the primary mechanism leading to limited pathophysiological changes result in oxidative stress, immune responses, fibrosis, apoptosis and cardiac dysfunction.

Previous studies have found that AMPK plays a key role in intracellular metabolism and is an attractive therapeutic target, and that switching on/off of AMPK will leads to changes in the expression of SIRT3[56]. There is currently no research on the link between NEU1 and AMPK. In agreement with this finding, we found that AMPKa deficiency abolishes the protection provided by shNEU1 against fibrosis, inflammation, apoptosis and oxidative stress. Our study further suggested that the inhibitory effects of shNEU1 against diabetes-related cardiac dysfunction were offset by AMPKa deficiency in vivo. Importantly, shNEU1 elevated SIRT3 expression was inhibited by AMPKa deficiency, declaring that AMPKa activation was responsible for shNEU1-mediated upregulation of SIRT3.

A critical issue raised by our data in this study relates to the activation of the AMPKa pathway by NEU1 inhibition. AMPK activation is mediated by upstream kinases (such as LKB1, CaMMK $\beta$, and TAK1)[26]. In this study, we found that siLKB1 blocked shNEU1-induced AMPKa activation. However, the observation that CaMMK $\beta$ inhibitor and TAK1 inhibitor did not affect AMPKa activation caused by shNEU1 suggested that CaMMK $\beta$ and TAK1 were not involved in the shNEU1-mediated AMPKa activation. These results further indicate that NEU1 inhibition activates AMPKa via LKB1 on diabetic cardiomyopathy. It has been reported that AMPK is activated through phosphorylation via LKB1 at the lysosome in an AMP-independent manner. LKB1 which was required for canonical energy sensing by AMPK [57]. We do not yet know the mechanisms of NEU1 on LKB1 activity; however, this determination will require extensive work that is beyond the scope of our study. The cellular targeting of LKB1 in the NRVM infected with shNEU1 is adequate for the points made in this manuscript: that the cell viability after high glucose is rescued, at least in part, by NEU1 inhibition, and that NRVM with LKB1 silence but not CaMMK $\beta$ inhibitor and TAK1 inhibiter, blocked the NEU1 inhibition effects in cell viability, as well as the expression of downstream signaling.

This study has some limitations. First, NEU1 was elevated in STZ-induced diabetic mice heart and in HG-stimulated cardiomyocytes. However, we did not explore why and how NEU1 increased, nor did we explore whether its expression in other tissues or cells changed, such as pancreatic islets. Second, we found that silencing NEU1 can activate AMPK in a high glucose environment through LKB1, but we have not further studied the specific mechanism by which NEU1 directly affects LKB1. Third, in this experiment, we mainly used AAV9 to verify its effect, and did not use genetically engineered mice related to NEU1. Fourth, in this study, only the effects of NEU1 were tested, and did not verify the expression and role of other NEUs (eg. NEU3). Fifth, this research did the exploration of NEU1 inhibition, but did not conduct the research of overexpression of NEU1.

In summary, the present study identified NEU1 deficiency attenuated STZ-induced diabetic cardiomyopathy. NEU1 inhibition activates AMPKa via LKB1, and subsequently activates sirt3, thereby regulating fibrosis, inflammation, apoptosis and oxidative stress in diabetic myocardial tissue. 


\section{Competing Interests}

The authors have declared that no competing interest exists.

\section{Supplementary Material}

Supplementary figures and tables. https://www.ijbs.com/v18p0826s1.pdf

\section{Acknowledgements}

\section{Data Availability}

Data supporting the findings of this study could be obtained from the corresponding author upon reasonable request.

\section{Funding Sources}

\section{National Natural Science Foundation of China (81900219, 81800216);}

\section{Author Contributions}

Z.G., H.T., N.T. and F.Y.L. performed experiments and analyzed the data. Z.G., D.F., Z.Y. and Q.Z.T. designed experiments. Z.G, H.T. and D.F. wrote and edited the manuscript. S.Q.M., P.A., D.Y., and M.Y.W. assisted with some experiments and discussed the results. Z.G., H.T., N.T. and F.Y.L. conducted bioinformatics analysis. Z.G., D.F., Z.Y. and Q.Z.T. supervised and conceptualized the study, wrote and edited the manuscript.

\section{References}

1. Poirier P, Bogaty P, Garneau C, Marois L, Dumesnil JG. Diastolic dysfunction in normotensive men with well-controlled type 2 diabetes: importance of maneuvers in echocardiographic screening for preclinical diabetic cardiomyopathy. Diabetes Care. 2001; 24: 5-10.

2. Kajstura J, Fiordaliso F, Andreoli AM, Li B, Chimenti S, Medow MS, et al IGF-1 overexpression inhibits the development of diabetic cardiomyopathy and angiotensin II-mediated oxidative stress. Diabetes. 2001; 50: 1414-24.

3. Pacher P, Beckman IS, Liaudet L. Nitric oxide and peroxynitrite in health and disease. Physiol Rev. 2007; 87: 315-424.

4. Lopaschuk GD, Ussher JR, Folmes CD, Jaswal JS, Stanley WC. Myocardial fatty acid metabolism in health and disease. Physiol Rev. 2010; 90: 207-58.

5. Herrero P, Peterson LR, McGill JB, Matthew S, Lesniak D, Dence C, et al. Increased myocardial fatty acid metabolism in patients with type 1 diabetes mellitus. J Am Coll Cardiol. 2006; 47: 598-604.

6. Min W, Bin ZW, Quan ZB, Hui ZJ, Sheng FG. The signal transduction pathway of PKC/NF-kappa B/c-fos may be involved in the influence of high glucose on the cardiomyocytes of neonatal rats. Cardiovasc Diabetol. 2009; 8: 8

7. Falcao-Pires I, Hamdani N, Borbely A, Gavina C, Schalkwijk CG, van der Velden J, et al. Diabetes mellitus worsens diastolic left ventricular dysfunction in aortic stenosis through altered myocardial structure and cardiomyocyte stiffness. Circulation. 2011; 124: 1151-9.

8. Ritchie RH, Quinn JM, Cao AH, Drummond GR, Kaye DM, Favaloro JM, et al. The antioxidant tempol inhibits cardiac hypertrophy in the insulin-resistant GLUT4-deficient mouse in vivo. J Mol Cell Cardiol. 2007; 42: 1119-28.

9. Hanson VA, Shettigar UR, Loungani RR, Nadijcka MD. Plasma sialidase activity in acute myocardial infarction. Am Heart J. 1987; 114: 59-63.

10. Zhang L, Wei TT, Li Y, Li J, Fan Y, Huang FQ, et al. Functional Metabolomics Characterizes a Key Role for N-Acetylneuraminic Acid in Coronary Artery Diseases. Circulation. 2018; 137: 1374-90.

11. Yuan YP, Ma ZG, Zhang X, Xu SC, Zeng XF, Yang Z, et al. CTRP3 protected against doxorubicin-induced cardiac dysfunction, inflammation and cell death via activation of Sirt1. J Mol Cell Cardiol. 2018; 114: 38-47.

12. Zhang M, Zhang L, Hu J, Lin J, Wang T, Duan Y, et al. MST1 coordinately regulates autophagy and apoptosis in diabetic cardiomyopathy in mice. Diabetologia. 2016; 59: 2435-47.

13. Xiao $Y$, Wu OQ Duan $M X$, Liu $C$, Yuan $Y$, Yang $Z$, et al. TAX1BP1 overexpression attenuates cardiac dysfunction and remodeling in
STZ-induced diabetic cardiomyopathy in mice by regulating autophagy. Biochim Biophys Acta Mol Basis Dis. 2018; 1864: 1728-43.

14. Chen W, Zheng R, Baade PD, Zhang S, Zeng H, Bray F, et al. Cancer statistics in China, 2015. CA Cancer J Clin. 2016; 66: 115-32.

15. Ren J, Zhang N, Liao H, Chen S, Xu L, Li J, et al. Caffeic acid phenethyl ester attenuates pathological cardiac hypertrophy by regulation of MEK/ERK signaling pathway in vivo and vitro. Life Sci. 2017; 181: 53-61.

16. Chen Y, Qing W, Sun M, Lv L, Guo D, Jiang Y. Melatonin protects hepatocytes against bile acid-induced mitochondrial oxidative stress via the AMPK-SIRT3-SOD2 pathway. Free Radic Res. 2015; 49: 1275-84.

17. Ma ZG, Dai J, Zhang WB, Yuan Y, Liao HH, Zhang N, et al. Protection against cardiac hypertrophy by geniposide involves the GLP-1 receptor / AMPKalpha signalling pathway. Br J Pharmacol. 2016; 173: 1502-16.

18. Ma ZG, Yuan YP, Xu SC, Wei WY, Xu CR, Zhang X, et al. CTRP3 attenuates cardiac dysfunction, inflammation, oxidative stress and cell death in diabetic cardiomyopathy in rats. Diabetologia. 2017; 60: 1126-37.

19. Totzke J, Gurbani D, Raphemot R, Hughes PF, Bodoor K, Carlson DA, et al. Takinib, a Selective TAK1 Inhibitor, Broadens the Therapeutic Efficacy of TNF-alpha Inhibition for Cancer and Autoimmune Disease. Cell Chem Biol. 2017; 24: 1029-39 e7.

20. Tanaka T, Iino M. Nuclear Translocation of p65 is Controlled by Sec6 via the Degradation of IkappaBalpha. J Cell Physiol. 2016; 231: 719-30.

21. Turkmen K, Karagoz A, Kucuk A. Sirtuins as novel players in the pathogenesis of diabetes mellitus. World J Diabetes. 2014; 5: 894-900.

22. Zhang HH, Ma XJ, Wu LN, Zhao YY, Zhang PY, Zhang YH, et al. Sirtuin-3 (SIRT3) protects pancreatic beta-cells from endoplasmic reticulum (ER) stress-induced apoptosis and dysfunction. Mol Cell Biochem. 2016; 420: 95-106.

23. Koentges C, Pfeil K, Schnick T, Wiese S, Dahlbock R, Cimolai MC, et al. SIRT3 deficiency impairs mitochondrial and contractile function in the heart. Basic Res Cardiol. 2015; 110: 36

24. Xie X, Wang L, Zhao B, Chen Y, Li J. SIRT3 mediates decrease of oxidative damage and prevention of ageing in porcine fetal fibroblasts. Life Sci. 2017; 177: 41-8.

25. Chen $Y$, Chen $C$, Dong B, Xing F, Huang H, Yao F, et al. AMPK attenuates ventricular remodeling and dysfunction following aortic banding in mice via the Sirt3/Oxidative stress pathway. Eur J Pharmacol. 2017; 814: 335-42.

26. Herrero-Martin G, Hoyer-Hansen M, Garcia-Garcia C, Fumarola C, Farkas T, Lopez-Rivas A, et al. TAK1 activates AMPK-dependent cytoprotective autophagy in TRAIL-treated epithelial cells. EMBO J. 2009; 28: 677-85.

27. Sieve I, Ricke-Hoch M, Kasten M, Battmer K, Stapel B, Falk CS, et al. A positive feedback loop between IL-1beta, LPS and NEU1 may promote atherosclerosis by enhancing a pro-inflammatory state in monocytes and macrophages. Vascul Pharmacol. 2018; 103-105: 16-28.

28. Heimerl M, Sieve I, Ricke-Hoch M, Erschow S, Battmer K, Scherr M, et al. Neuraminidase-1 promotes heart failure after ischemia/reperfusion injury by affecting cardiomyocytes and invading monocytes/macrophages. Basic Res Cardiol. 2020; 115: 62.

29. Yang A, Gyulay G, Mitchell M, White E, Trigatti BL, Igdoura SA. Hypomorphic sialidase expression decreases serum cholesterol by downregulation of VLDL production in mice. J Lipid Res. 2012; 53: 2573-85.

30. Saito M, Sakiyama K, Shiota $T$, Ito $M$. Isoproterenol produces a rapid increase in sialidase activity in rat heart tissue and cardiomyocyte-derived $\mathrm{H} 9 \mathrm{c} 2$ cells in culture. FEBS Lett. 2003; 542: 105-8.

31. Chen OQ, Ma G, Liu JF, Cai YY, Zhang JY, Wei TT, et al. Neuraminidase 1 is a driver of experimental cardiac hypertrophy. Eur Heart J. 2021; 42: 3770-82.

32. Pulicherla N, Shen S, Yadav S, Debbink K, Govindasamy L, Agbandje-McKenna M, et al. Engineering liver-detargeted AAV9 vectors for cardiac and musculoskeletal gene transfer. Mol Ther. 2011; 19: 1070-8.

33. Zeng H, Vaka VR, He X, Booz GW, Chen JX. High-fat diet induces cardiac remodelling and dysfunction: assessment of the role played by SIRT3 loss. J Cell Mol Med. 2015; 19: 1847-56.

34. Alrob OA, Sankaralingam S, Ma C, Wagg CS, Fillmore N, Jaswal JS, et al. Obesity-induced lysine acetylation increases cardiac fatty acid oxidation and impairs insulin signalling. Cardiovasc Res. 2014; 103: 485-97.

35. He X, Zeng H, Chen JX. Emerging role of SIRT3 in endothelial metabolism, angiogenesis, and cardiovascular disease. J Cell Physiol. 2019; 234: 2252-65.

36. Yu W, Gao B, Li N, Wang J, Qiu C, Zhang G, et al. Sirt3 deficiency exacerbates diabetic cardiac dysfunction: Role of Foxo3A-Parkin-mediated mitophagy. Biochim Biophys Acta Mol Basis Dis. 2017; 1863: 1973-83.

37. Ye W, Tang X, Yang Z, Liu C, Zhang X, Jin J, et al. Plasma-derived exosomes contribute to inflammation via the TLR9-NF-kappaB pathway in chronic heart failure patients. Mol Immunol. 2017; 87: 114-21.

38. Sultana MR, Bagul PK, Katare PB, Anwar Mohammed S, Padiya R, Banerjee SK. Garlic activates SIRT-3 to prevent cardiac oxidative stress and mitochondrial dysfunction in diabetes. Life Sci. 2016; 164: 42-51.

39. Hou X, Zeng H, He X, Chen JX. Sirt3 is essential for apelin-induced angiogenesis in post-myocardial infarction of diabetes. J Cell Mol Med. 2015; 19: 53-61.

40. Vadvalkar SS, Matsuzaki S, Eyster CA, Giorgione JR, Bockus LB, Kinter CS, et al. Decreased Mitochondrial Pyruvate Transport Activity in the Diabetic Heart: ROLE OF MITOCHONDRIAL PYRUVATE CARRIER 2 (MPC2) ACETYLATION. J Biol Chem. 2017; 292: 4423-33.

41. Yang W, Nagasawa K, Munch C, Xu Y, Satterstrom K, Jeong S, et al. Mitochondrial Sirtuin Network Reveals Dynamic SIRT3-Dependent 
Deacetylation in Response to Membrane Depolarization. Cell. 2016; 167: 985-1000 e21.

42. Hirschey MD, Shimazu T, Goetzman E, Jing E, Schwer B, Lombard DB, et al. SIRT3 regulates mitochondrial fatty-acid oxidation by reversible enzyme deacetylation. Nature. 2010; 464: 121-5.

43. Zhou Y, Chung ACK, Fan R, Lee HM, Xu G, Tomlinson B, et al. Sirt3 Deficiency Increased the Vulnerability of Pancreatic Beta Cells to Oxidative Stress-Induced Dysfunction. Antioxid Redox Signal. 2017; 27: 962-76.

44. Giralt A, Villarroya F. SIRT3, a pivotal actor in mitochondrial functions: metabolism, cell death and aging. Biochem J. 2012; 444: 1-10.

45. Dikalova AE, Itani HA, Nazarewicz RR, McMaster WG, Flynn CR, Uzhachenko R, et al. Sirt3 Impairment and SOD2 Hyperacetylation in Vascular Oxidative Stress and Hypertension. Circ Res. 2017; 121: 564-74.

46. Dikalov $\mathrm{S}$, Itani $\mathrm{H}$, Richmond B, Vergeade A, Rahman SMI, Boutaud O, et al. Tobacco smoking induces cardiovascular mitochondrial oxidative stress, promotes endothelial dysfunction, and enhances hypertension. Am J Physiol Heart Circ Physiol. 2019; 316: H639-H46.

47. Someya S, Yu W, Hallows WC, Xu J, Vann JM, Leeuwenburgh C, et al. Sirt3 mediates reduction of oxidative damage and prevention of age-related hearing loss under caloric restriction. Cell. 2010; 143: 802-12.

48. Rathore R, Zheng YM, Niu CF, Liu QH, Korde A, Ho YS, et al. Hypoxia activates NADPH oxidase to increase [ROS]i and $[\mathrm{Ca} 2+]$ i through the mitochondrial ROS-PKCepsilon signaling axis in pulmonary artery smooth muscle cells. Free Radic Biol Med. 2008; 45: 1223-31.

49. Dikalov S. Cross talk between mitochondria and NADPH oxidases. Free Radic Biol Med. 2011; 51: 1289-301.

50. Jia G, DeMarco VG, Sowers JR. Insulin resistance and hyperinsulinaemia in diabetic cardiomyopathy. Nat Rev Endocrinol. 2016; 12: 144-53.

51. Psefteli PM, Kitscha P, Vizcay G, Fleck R, Chapple SJ, Mann GE, et al. Glycocalyx sialic acids regulate Nrf2-mediated signaling by fluid shear stress in human endothelial cells. Redox Biol. 2021; 38: 101816.

52. Wang J, Song Y, Wang Q, Kralik PM, Epstein PN. Causes and characteristics of diabetic cardiomyopathy. Rev Diabet Stud. 2006; 3: 108-17.

53. Abdulkhalek S, Amith SR, Franchuk SL, Jayanth P, Guo M, Finlay T, et al. Neu1 sialidase and matrix metalloproteinase-9 cross-talk is essential for Toll-like receptor activation and cellular signaling. J Biol Chem. 2011; 286: 36532-49.

54. Amith SR, Jayanth P, Franchuk S, Finlay T, Seyrantepe V, Beyaert R, et al. Neu1 desialylation of sialyl alpha-2,3-linked beta-galactosyl residues of TOLL-like receptor 4 is essential for receptor activation and cellular signaling. Cell Signal. 2010; 22: 314-24.

55. Stamatos NM, Liang F, Nan X, Landry K, Cross AS, Wang LX, et al. Differential expression of endogenous sialidases of human monocytes during cellular differentiation into macrophages. FEBS J. 2005; 272: 2545-56.

56. Yu X, Zhang L, Yang X, Huang H, Huang Z, Shi L, et al. Salvianolic acid A protects the peripheral nerve function in diabetic rats through regulation of the AMPK-PGC1alpha-Sirt3 axis. Molecules. 2012; 17: 11216-28.

57. Lin SC, Hardie DG. AMPK: Sensing Glucose as well as Cellular Energy Status. Cell Metab. 2018; 27: 299-313. 